\title{
The Determination of Surface Thermodynamic Properties of Nanoparticles by Thermal Analysis
}

\author{
George O. Piloyan, Nikolay S. Bortnikov, Natalia M. Boeva \\ Institute of Geology of Ore Deposits, Petrography, Mineralogy and Geochemistry, \\ Russian Academy of Sciences (IGEM RAS), Moscow, Russia \\ Email: bns@igem.ru, boeva@igem.ru
}

Received April 23, 2013; revised May 26, 2013; accepted June 29, 2013

Copyright (C) 2013 George O. Piloyan et al. This is an open access article distributed under the Creative Commons Attribution License, which permits unrestricted use, distribution, and reproduction in any medium, provided the original work is properly cited.

\begin{abstract}
The effect of dispersivity on thermodynamic and kinetic parameters of chemical reactions in nanodispersed systems is theoretically investigated. On the basis of the established theoretical dependences the new method of determination of surface thermodynamic properties of nanoparticles (surface enthalpy, surface entropy and surface energy) by thermal analysis (DTA or DSC) was developed. Three examples of calculation of surface properties of nanoparticles were presented to prove the feasibility of this method.
\end{abstract}

Keywords: Surface Enthalpy; Surface Entropy; Surface Energy; DTA; DSC; Nanoparticle

\section{Introduction}

Surface thermodynamic properties of nanoparticles take a distinct effect on thermodynamic and kinetic parameters of chemical reactions (so-called size effect) in nanodyspersed systems. However, this problem is poorly investigated because of complexity of experiment and absence of a database on surface thermodynamic properties of solid. The present article attempts to fill an existing gap somewhat. In the article, the new method of determination of surface thermodynamic properties of nanoparticles by thermal analysis is described and its influence on thermodynamic and kinetic parameters of chemical reaction is shown.

Owing to its great scientific and practical significance, the influence of solid body dispersibility on the chemical reaction velocity has attracted the attention of researchers for a long time. Three different kinetic models, which take into account substance dispersibility, were proposed for different heterogeneous chemical reactions [1]. At the same time, all the models assume that changes in dispersibility affect only the size of the reactive surface in the solid body. It was shown that the substance dispersibility growth is accompanied by a shift of the reaction regime from the diffusion-kinetic area toward the kinetic one [2]. The processes at the phase interface form the limiting stage of kinetics.

The transition to nanoparticles is characterized by size effects. It is established that the thermal effect of the re- action Qr for nanoparticles depends on the surface energy $\sigma[3]$.

\section{About Terminology}

Let's define some terms which will be used in the further.

\subsection{Nanoparticle}

As this term we shall understand particles of substance which size even in one dimension lay in an interval 10 $\mathrm{nm} \leq r 100 \mathrm{~nm}$ [4]. In other words, nanoparticles are larger clusters, but it is less than microcrystals. Under this definition get also a number of natural substances (some clay minerals, some oxides, etc.). The main characteristic property of nanopartiles is the appreciable contribution of surface energy to their total energy.

\subsection{Nanodispersed System}

We shall understand as this term the system which consists of nanoparticles.

\subsection{Surface Energy, Surface Tension}

Energetic properties of a surface of solid is usually characterized value of a surface tension $\gamma$ (by analogy to liquids) $\left(\mathrm{J} / \mathrm{m}^{2}\right)$ or surface energy $\sigma\left(\mathrm{J} / \mathrm{m}^{2}\right)$.

The surface tension is caused by unbalanced field of 
intermolecular forces, i.e., by definition, it is value isotropic. The condition is satisfied always for liquids. An alternative pattern is observed for solid. Stress tensors in volume and in surface layer of solid are not isotropic in general so also the surface tension should be not obligatory isotropic, that contradicts its definition. Thus, the concept of a surface tension for a solid has concrete physical sense only within the range of melting temperature of a solid.

More universal concept is surface energy. There is a simple thermodynamic relationship between a surface tension and surface energy for liquid [1]:

$$
\gamma=\sigma+F(\partial \sigma / \partial F)
$$

where $F$ is area of surface of liquid.

For pure liquids (one-component system) $\partial \sigma / \partial F=0$, so numerically $\gamma=\sigma$.

According to Gibbs, thermodynamic properties of nanopartiles is described the same thermodynamic functions, as microcrystals. For example, Gibbs free energy of nanoparticle $G_{r}$ is stated as follows:

$$
G_{r}=G_{\infty}+\sigma F,
$$

where $G_{\infty}$ - Gibbs free energy of microcrystal, $F$ - the area of a surface of nanoparticle, $\sigma$ is surface energy of nanoparticle:

$$
\sigma=H_{F}-T S_{F}
$$

where $H_{F}$-surface enthalpy, $S_{F}$-surface entropy, $T$ is temperature. For pure substances (one-component systems) $H_{F}>0, S_{F}>0, H_{F}$ and $S_{F}$ do not depend on temperature as a first approximation [1]. $H_{F}$ is often named surface energy or total surface energy to suppose approximately the equality $\sigma \approx H_{F}$ for nanoparticles at first approximation.

\section{The Offered Model}

Let investigated substance represents ensemble independent nanoparticles, contacting, but not cooperating with each other. We shall admit, that everyone nanoparticle represents pure substance (one-component system). The total area of a surface of ensemble nanoparticles we shall characterize the specific area $\mathrm{F}$ carried to 1 mole substances $\left(\mathrm{m}^{2} / \mathrm{mol}\right)$ :

$$
F=(S c) / \rho r,
$$

where $S c$ is shape coefficient ( $S c=3$ for spherical particles, $S c=2$ for lamellar particles, $S c=2.5$ for particles of the complex or uncertain form, etc.), $\rho-$ mole density of a particle, $r$ - the characteristic size of a particle.

Let chemical reaction occur in the system:

$$
A_{S}+v_{B} B_{g a s}=v_{C} C_{S}+v_{D} D_{g a s}
$$

where $v_{i}$ are stoichiometric coefficients, $v_{A}=1$, i.e. all calculations are related to a mole of the initial substance.

The assumption, that the transformation process in a system can be described by one chemical reaction, is equivalent to an assumption, which this process depends on one independent variable. The number of moles of any reaction component can be selected as such a variable, but is more convenient to introduce a new variable $\alpha$ which is known as the degree of transformation or fractional extent of reaction.

According to the definition:

$$
\alpha=\left(n_{0 i}-n_{i}\right) / v_{i} n_{0 i},
$$

$\alpha$ is dimensionless quantity, $0 \leq \alpha \leq 1$.

If we assume, that all components of system mutually insoluble, reaction in system should go up to the end $(\alpha=1)$, unless one of the components is exhausted.

Hence, nanopartile $A$ is changed into nanoparticle $\tilde{N}$ as a result of reaction (5) according to our model.

\section{Some Features of Thermodynamics of Chemical Reactions}

For our model Gibbs free energy of reaction $\Delta G_{1}$ is determined as:

$$
\Delta G_{r}=\sum_{i=1}^{n} G_{i} v_{i}
$$

where $G_{i}$ is thermodynamic potential of component $i, v_{i}$, are stoichiometric coefficients, $v_{i}<0$ for reagents and $v_{i}>0$ for the reaction products.

In the equilibrium state, we have

$$
\Delta G_{r}=0
$$

Let's consider separate types of transformations, described by Equation (5).

$$
\text { Phase transitions }\left(v_{B}=v_{D}=0, v_{C}=1\right) \text {. }
$$

With account for (2) and (5), we can write the following equation:

$$
\Delta G_{r}=\Delta G_{\infty}+\sigma_{C} F_{C}-\sigma_{A} F_{A} .
$$

Taking into account equality $G=H-T S$, constancy of mass and (4), (8), we get (for spherical particles):

$$
Q_{\infty} \frac{\Delta T}{\left(T_{0}\right)_{\infty}}=\frac{3}{\rho r}\left(\sigma_{A}-\sigma_{C}^{*}\left(\frac{\rho_{C}}{\rho_{A}}\right)^{\frac{2}{3}}\right),
$$

where $Q_{\infty}$ is heat of phase transition related to the microcrystalline state, $\Delta T=\left(T_{0}\right)_{\infty}-T_{0}$ is equilibrium temperature difference between temperatures of phase transitions for the microcrystalline and nanodispersed state of the substance, $\rho$ is the mole density of nanoparticles and 
$r$ is the size of nanoparticles.

Formula (10) for the first time has been derived by Hill [5]. This formula can be considered as the generalized analogue of known Gibbs - Thomson formula.

For heterogeneous endothermic (or exothermic) reactions the equation is obtained similar to Equation (9):

$$
\Delta G_{r}=\left(\Delta G_{r}\right)_{\infty}+v_{C} \sigma_{C} F_{C}-\sigma_{A} F_{A} .
$$

In the equilibrium state, we have

$$
\Delta G_{r}=0
$$

A little manipulation yields as follows (for spherical particles):

$$
Q_{\infty} \frac{\Delta T}{\left(T_{0}\right)_{\infty}}=\frac{3 M_{A}}{\rho_{A} r_{A}}\left[\sigma_{A}-\sigma_{C}\left(\frac{v_{C} M_{C}}{M_{A}} \frac{\rho_{A}}{\rho_{C}}\right)^{\frac{2}{3}}\right],
$$

where $M_{i}$ are the molecular masses of nanoparticles $A$ and $C, \rho_{i}$ are their densities. Complex $\left(v_{C} M_{C} / M_{A}\right)$ is known as Pilling-Bedward coefficient in the literature [6].

\section{Some Features of Kinetics of Chemical Reactions in the Nanodispersed Systems}

The effect dispersivity of a solid on the chemical reactions rate has attracted the attention of researches for a long time, owing to its great scientific and practical significance. The various kinetic models, which take into account dispersivity, were proposed for different chemical reactions [2].

However in all models it is supposed, that the change of dispersivity changes only the area of a reactionary surface of a solid. The dispersivity growth has been shown to be accompanied by a shift of the reaction regime from the diffusion-kinetic area toward the kinetic one [7]. A limiting kinetic stage becomes the processes going on surface of the interface. In this case, reaction rate depends on value of the surface and can be presented Equation [2]:

$$
\frac{\mathrm{d} V_{t}}{\mathrm{~d} t}=k F_{t},
$$

where $V_{t}$ and $F_{t}$ are accordingly volume and a surface of the particle, not reacted by the moment $t, k$ is the rate constant.

By introducing degree of transformation $\alpha$ (5) into Equation (14), we obtain following equation:

$$
\frac{\mathrm{d} \alpha}{\mathrm{d} t}=\left(k / r_{0}\right)(1-\alpha)^{n}
$$

where $n$ is the order of reaction (for particles of the spherical form $n=2 / 3$, for flat $n=1 / 2$, etc. ), $r_{0}$ is the size of the particle.

The rate constant $\mathrm{k}$ is usually described by the Ar- rhenius equation:

$$
k=A \exp (-E / R T)
$$

where $A$ is a pre-exponential term, $E$ is empirical (apparent) energy of activation, $R$ is a gas constant, $T$ is temperature in Kelvin.

In the theory of the activated complex (one of the basic theories of chemical kinetics) [8], the rate constant is defined by the equation:

$$
k=A_{1} \exp \left(-G_{0} / R T\right),
$$

where $A_{1}=\chi\left(k_{B} T / h\right), \quad k_{B}$ is Boltzmann's constant, $h$ is Planck's constant, $G_{0}$ is activation free energy, $\chi$ is transmission coefficient. $\chi$ defines a probability that the system to jump activation barrier. It usually is assumed that $\chi=1$.

Equation (17) for nanoparticle must be changed by analogy with Equation (9). According to work [9] the rate constant of reaction of the activated complex which has already formed on a surface nanoparticle, should not depends on dispersivity. According to this assumption Equation (9) may be transformed as follows:

$$
\Delta G_{r}=G_{0}-G_{A r}=G_{0}-G_{A \infty}-\sigma_{A} F_{A}=\Delta G_{0}-\sigma_{A} F_{A}
$$

where $G_{A r}$ is free energy initial nanoparticle $A, G_{A \infty}$ is Gibbs free energy for microcrystals of an initial component $A, \Delta G_{0}$ is free energy of formation of the activated complex.

Thus Formula (18) may be written as follows:

$$
k=A_{1} \exp \left(-\left(G_{0}-\sigma F\right)\right) / R T
$$

where $\sigma$ is surface energy, $F$ is the mole area of surface.

Formula (19) can be transformed taking into account equality (3):

$$
k=A_{2} \exp \left(-\left(H_{0}-\sigma F\right)\right) / R T
$$

where $H_{0}$ is activation enthalpy, $A_{2}=A_{1} \exp \left(S_{0} / R\right)$, $S_{0}$ is activation entropy.

The following equation has been derived in the theory of the activated complex [7]:

$$
H_{0}=E-R T
$$

where $E$ is activation energy of reaction for microcrystalls.

Having substituted (21) in (20) we shall receive:

$$
k=A_{0} \exp (-(E-\sigma F)) / R T
$$

where $A_{0}=e A_{2}$,

Substitution in (15) Formulas (20) and (21) gives:

$$
\frac{\mathrm{d} \alpha}{\mathrm{d} t}=\left(A_{0} / r_{0}\right) \exp (-(E-\sigma F)) / R T(1-\alpha)^{n}
$$

From Equation (23) we may deduce that rate of 
chemical reaction should increase with growth of dispersivity.

Of course, this is an idealized situation, which takes into account neither defects nor the covering degree of active centers on the reactive surface of the substance.

A more general example of heterogeneous catalytic reactions is considered in [9], where it is shown that the dispersivity growth under stationary filling of active centers of the catalyzing agent $\theta>1$, the activation energy should decrease, while under $\theta<1$, the latter should grow.

Below it will be shown, what even the simplified model considered in the article, leads to satisfactory results.

\section{Some Formulas from the Theory of the Thermal Analysis}

The thermal analysis experiments are known to run in non-isothermal conditions. According to the theory of the thermal analysis [10], parameters of thermal curves (DTA, DSC, TG, DTG) contains the usefulness information on investigated substance and processes, in it proceeding.

For the decision of our problem it is enough to use only one parameter - peak temperature of the thermal effect (temperature of the maximal deviation of thermal curves from a base line in an interval of thermal effect).

The peak temperature of curve DTA, DTG or DSC is usually assumed to correspond to the temperature of maximal rate of chemical reaction. This assumption can be accepted only in the case where inertia of balance for DTG curves or thermal inertia of substance for curves DTA or DSC may neglect.

It has theoretically been shown in work [11], that the peak temperature on curves DTG should advance peak temperature on curves DTA for endothermic reactions.

However, practice shows, that this difference is usually insignificant, so as a first approximation it is possible to accept, that for curves DTA or DSC the peak temperature $T_{m}$ of endothermic effect is approximately equal to temperature of maximal rate reaction. Thus, at $T \rightarrow T_{m}$ the equation is accomplished:

$$
\frac{\mathrm{d}^{2} \alpha}{\mathrm{d} t^{2}}=0
$$

More complex picture, especially for fast reactions with greater heat effect, is observed for exothermic reactions.

Let's consider endothermic reaction of the first order. Twice differentiating (15) with respect to $t$ we shall receive:

$$
\left(E / R T^{\wedge} 2\right) d T / d t=A \exp (-E / R T) / r_{0}
$$

It is known [10], that for curves DTA or DSC at $T \rightarrow T_{m}$

$$
\mathrm{d} T / \mathrm{d} t \rightarrow b,
$$

where $b$ is heating rate of the furnace or reference sample.

For curves DTG at $T \rightarrow T_{m}$ the derivative $\mathrm{d} T / \mathrm{d} t \neq b$. For this reason the use of curves DTA or DSC is more preferable, as they contain less unknown parameters than curves DTG.

In [12] it has been shown, that at $T \rightarrow T_{m}$ as a first approximation:

$$
n(1-\alpha)^{(n-1)} \rightarrow 1
$$

Hence, Equation (24) can be used and for reactions of the $n$-order.

If instead of $k$ from (16) to use $k$ from (22) we shall receive:

$$
r_{0} b(E-\sigma F) / R T^{\wedge} 2=\left(A_{0} \exp (-(E-\sigma F) / R T)\right) .
$$

For convenience, Formula (27) transforms more convenient form:

$$
r_{0}=\left[A_{0}\left(R T^{\wedge} 2\right) / b(E-\sigma F)\right] \exp (-(E-\sigma F)) / R T .
$$

Denote the term in a square brackets by $B$. Equation (28) may be rewritten as follows:

$$
r_{0}=B \exp (-(E-\sigma F) / R T) .
$$

At change $T$ or $r$ term $B$ changes essentially more slowly, than the exponential term. As a first approximation the term $B$ may be assumed to be constant:

$$
B=\text { const. }
$$

Write down (29) in form more convenient for calculation, having substituted instead of $F$ and $\sigma$ their values from Formulas (3) and (4):

$$
\begin{aligned}
\ln (r)= & \ln (B)-E / R T_{m}+(S c) H_{F} / \rho R r T_{m} \\
& -(S c) S_{F} / \rho R r,
\end{aligned}
$$

where $r$ is the size of a particle, $E$ is empirical activation energy for microcrystals, $T_{m}$ is peak temperature of thermal effects on curves DTA or DSC, $R$ is a gas constant, $(S c)$-shape factor, $H_{F}$ is surface enthalpy, $\rho$ is a mole density of substance, $S_{F}$ is surface entropy.

Thus, the value $\ln (r)$ depends on three independent variables $-1 / T_{m}, 1 / r T_{m}$ and $1 / r$. Coefficients at these variables from Equation (31) is calculated by method of multiple regression. The admissible calculation accuracy can be achieved if to use not less than 6 experimental points.

\section{The Calculations}

Let's consider on the several examples, how much well offered model describes experimental data. 
The published experimental data of different authors (see Table 1) have been used as initial data.

The first example is related to dehydration of boehmite. $(\alpha-\mathrm{AlOOH})$. This reaction is known to belong to the class of topochemical reactions; i.e., their limiting stage is represented by processes at the phase interface. The rate reaction is described by Equation (23).

The authors [14] synthesized boehmite nanoparticles 1 to $26 \mathrm{~nm}$ across and described their thermal curves obtained on the differential scanning calorimeter (DSC). The size of particles was defined by X-ray method. Their experimental data are presented in Table 1. Using the boehmite data from Table 1, the multiple regression equation was calculated:

$$
\begin{aligned}
\ln (r)= & 18.6751-12366.1\left(1 / T_{m}\right)+3.37732\left(1 / r T_{m}\right) \\
& -0.0049445(1 / r)
\end{aligned}
$$

The coefficient $R^{2}=99.68 \%$.

Using the coefficients from Equation (31), we obtain (see Table 2 that the activation energy of the boehmite dehydration reaction is $E=102.8 \mathrm{~kJ} / \mathrm{mole}$, the surface enthalpy is $0.58 \mathrm{~J} / \mathrm{m}^{2}$, surface entropy is $0.000844 \mathrm{~J} /$ $\left(\mathrm{m}^{2} \cdot \mathrm{K}\right)$, surface energy is calculated at $298 \mathrm{~K} \sigma_{298}=$ $0.329 \mathrm{~J} / \mathrm{m}^{2}$, using Formula (3). At calculation shape coefficient has been put $(S c)=2.5$ as synthesized boehmite nanoparticles have the uncertain forms. In the literature there are data on the surface enthalpy of boehmite, obtained by a method high-temperature calorimetry [15]: $H_{F}=0.52 \mathrm{~J} / \mathrm{m}^{2}$. Our data are fairly consistent with literary data.

The second example is related to the oxidation of magnetite.

\begin{tabular}{|c|c|c|c|c|c|c|}
\hline \multicolumn{2}{|c|}{$\gamma-\mathrm{Al}_{2} \mathrm{O}_{3}$} & \multicolumn{2}{|c|}{ Magnetite $\mathrm{Fe}_{3} \mathrm{O}_{4}$} & \multicolumn{2}{|c|}{ Boehmite $\alpha$-AlOOH } & \multirow[t]{2}{*}{ Sample } \\
\hline$r$ & $\mathrm{Tm}$ & $r$ & $\mathrm{Tm}$ & $r$ & $\mathrm{Tm}$ & \\
\hline 2.65 & 1463 & 9.5 & 358 & 1.13 & 653 & \\
\hline 2.69 & 1471 & 16 & 378 & 1.56 & 676 & \\
\hline 3 & 1476 & 30 & 388 & 2.04 & 686 & \\
\hline 3.3 & 1479 & 44 & 398 & 2.42 & 701 & \\
\hline 4.5 & 1522 & 48 & 418 & 6.9 & 744 & \\
\hline 6.2 & 1562 & 60 & 433 & 14.2 & 781 & \\
\hline 6.6 & 1563 & 80 & 433 & 26.3 & 801 & \\
\hline - & - & 95 & 438 & - & - & \\
\hline \multicolumn{2}{|c|}{$\begin{array}{l}\text { Phase tansformation } \\
\gamma-\mathrm{Al}_{2} \mathrm{O}_{3} \rightarrow \alpha-\mathrm{Al}_{2} \mathrm{O}_{3}\end{array}$} & \multicolumn{2}{|c|}{$\begin{array}{l}\text { Oxidation, the } \\
\text { formation } \\
\gamma-\mathrm{Fe}_{2} \mathrm{O}_{3}\end{array}$} & \multicolumn{2}{|c|}{$\begin{array}{l}\text { Dehydration, the } \\
\text { formation of } \gamma-\mathrm{Al}_{2} \mathrm{O}_{3}\end{array}$} & The reaction \\
\hline \multicolumn{2}{|c|}{ [13] } & \multicolumn{2}{|c|}{ [14] } & \multicolumn{2}{|c|}{ [13] } & References \\
\hline
\end{tabular}

Table 1. Initial experimental data.

The note: $\mathrm{Tm}$ is taken in $\mathrm{K}, r$ is taken in $\mathrm{nm}$.
Table 2. The results of calculation of surface thermodynamic properties of investigated substances.

\begin{tabular}{ccccccc}
\hline Sample & $\begin{array}{c}E, \\
\mathrm{~kJ} / \mathrm{mol}\end{array}$ & $\begin{array}{c}H_{F}, \\
\mathrm{~J} / \mathrm{m}^{2}\end{array}$ & $S_{F}, \mathrm{~J} / \mathrm{m}^{2} \mathrm{~K}$ & $\begin{array}{c}\sigma_{298}, \\
\mathrm{~J} / \mathrm{m}^{2}\end{array}$ & $\begin{array}{c}\rho, \\
\mathrm{g} / \mathrm{cm}^{3}\end{array}$ & $(S c)$ \\
\hline Boehmite & 102.8 & 0.58 & 0.0008437 & 0.329 & 3.08 & 2.5 \\
Magnetite & 16.1 & 1.85 & 0.00567 & 0.16 & 5.2. & 3 \\
$\gamma-\mathrm{Al}_{2} \mathrm{O}_{3}$ & 95.1 & 1.9 & 0.00144 & 1.474 & 3.6 & 3 \\
\hline
\end{tabular}

The note: $E$ is activation energy of chemical reaction for microcrystals, $H_{F}$ is surface enthalpy, $S_{F}$ is surface entropy, $\sigma_{298}$ - surface energy at $T$ $=298 \mathrm{~K}, \rho$ - density of substance, $(S c)$ - factor of the form.

Several kinetic models of oxidation reaction were proposed for many classes of solid substances [6,15]. All of them describe, however, processes for microcrystalline substances. The change-over to nanoparticles alters the picture of reaction. The limiting stage of reaction, as well as in case of with boehmite, becomes the process going on at the phase interface. Reaction rate is described by Equation (22). The authors of [14] synthesized magnetite nanoparticles 9.5 to $95 \mathrm{~nm}$ across and investigated oxidation reaction of magnetite and formation $\gamma$ $\mathrm{Fe}_{2} \mathrm{O}_{3}$ by DSC. The size of particles was determined by $\mathrm{X}$-ray method. Their experimental data are listed in Table 1. Using a calculation procedure similar to that in the situation with boehmite, we obtain the following multiple regression equation:

$$
\begin{aligned}
\ln (r)= & 9.39271-1937.36\left(1 / T_{m}\right)+29.7373\left(1 / r T_{m}\right) \\
& -0.091178(1 / r)
\end{aligned}
$$

The coefficient $R^{2}=99.53 \%$.

Using coefficients from Equation (31), we obtain that the activation energy of the magnetite oxidation reaction is $E=16.1 \mathrm{~kJ} / \mathrm{mole}$, the surface enthalpy is $H_{F}=1.85$ $\mathrm{J} / \mathrm{m}^{2}$, surface entropy is $S_{F}=0.00567 \mathrm{~J} /\left(\mathrm{m}^{2} \cdot \mathrm{K}\right)$. The surface energy under $298 \mathrm{~K}$ calculated in line with Equation (3) is $\sigma=0.16 \mathrm{~J} / \mathrm{m}^{2}$ (Table 2).

No data on magnetite surface properties are available in the literature. Let us use for the theoretical assessment of the surface enthalpy the Orovan equation [16], which allows the surface enthalpy for metals and some oxides to be calculated at first approximation:

$$
H_{F}=0.01 E_{y} a_{0}
$$

where $E_{y}$ is Young module and $a_{0}$ is the parameter of the lattice. Using the available published data $E_{y}=231.3$ $\mathrm{gPa}$ [17], $a_{0}=0.8394 \mathrm{~nm}$ [18], we obtain $H_{F}=1.941$ $\mathrm{J} / \mathrm{m}^{2}$. Quite satisfactory agreement to our result is observed.

The third example is related to the phase transformation $\gamma-\mathrm{Al}_{2} \mathrm{O}_{3} \rightarrow \alpha-\mathrm{Al}_{2} \mathrm{O}_{3}$.

In work [13] $\gamma-\mathrm{Al}_{2} \mathrm{O}_{3}$ has been synthesized at dehydration of boehmite nanoparticles. The size of particles was determined by X-ray method. Thermal curves DSC have 
been written down. Necessary for the further calculations the initial data are placed in Table 1. Using already described procedure of calculation we has been obtained the following multiple regression equation:

$$
\begin{aligned}
\ln (r)= & 9.8997-11442.90\left(1 / T_{m}\right)+19.468\left(1 / r T_{m}\right) \\
& -0.014763(1 / r)
\end{aligned}
$$

The coefficient $R^{2}=99.98 \%$.

Using coefficients from Equation (31), we obtain that the activation energy of the phase transformation $E$ is $95.1 \mathrm{~kJ} / \mathrm{mole}$, the surface enthalpy $\gamma-\mathrm{Al}_{2} \mathrm{O}_{3}$ is $=1.90 \mathrm{~J} / \mathrm{m}^{2}$, surface entropy $\gamma-\mathrm{Al}_{2} \mathrm{O}_{3}$ is $S_{F}=0.00144 \mathrm{~J} /\left(\mathrm{m}^{2} \cdot \mathrm{K}\right)$. The surface energy under $298 \mathrm{~K}$ calculated in line with Equation (3) is $\sigma=1.47 \mathrm{~J} / \mathrm{m}^{2}$ (Table 2).

In the literature there are data on the surface enthalpy of $\gamma-\mathrm{Al}_{2} \mathrm{O}_{3}$, obtained by the method high-temperature calorimetry [19]: $H_{F}=1.7 \mathrm{~J} / \mathrm{m}^{2}$. Our data are fairly consistent with literary data.

\section{Conclusions}

The basic equation of the method has been received:

$$
\begin{aligned}
\ln (r)= & \ln (B)-E / R T_{m}+(S c) H_{F} / \rho R r T_{m} \\
& -(S c) S_{F} / \rho R r
\end{aligned}
$$

where $r$ is the size of a particle, $E$ is empirical activation energy for microcrystals, $T_{m}$ is peak temperature on curves DTA or DSC, $R$ is a gas constant, $(S c)$ is shape coefficient, $H_{F}$ is surface enthalpy, $\rho$ is mole density of substance, $S_{F}$ is surface entropy.

$B=A_{0}\left(R T^{2}\right) / b(E-\sigma F), b$-heating rate of the reference substance or the furnace, $\ln (B) \approx$ const.

The equation involves one independent variable $\ln (r)$ and three dependent variables $1 / T_{m}, 1 / r T_{m}$ and $1 / r$. Coefficients in the equations are calculated by the multiple regression method. Surface energy is calculated by the known equation $\sigma=H_{F}-T S_{F}$. On an example of three reactions (dehydration of boehmite $(\alpha-\mathrm{AlOOH})$, oxidations of magnetite $\left(\mathrm{Fe}_{3} \mathrm{O}_{4} \rightarrow \gamma-\mathrm{Fe}_{2} \mathrm{O}_{3}\right)$ and phase transition $\left(\gamma-\mathrm{Al}_{2} \mathrm{O}_{3} \rightarrow \alpha-\mathrm{Al}_{2} \mathrm{O}_{3}\right)$ methods of calculation of surface properties were shown. The obtained results were compared with literature data. Our results quite well coincide with known literary data.

Three examples of calculation of surface properties of nanoparticles for three types of reactions show, what even for such simplified model which is accepted in present paper, it is possible to receive quite satisfactory results by thermal analysis.

\section{REFERENCES}

[1] Yu. G. Frolov, "Course of Colloid Chemistry," Khimiya, Moscow, 1982.

[2] Yu. D. Tret'yakov, "Solid Phase Reactions," Khimiya, Moscow, 1978.

[3] G. O. Piloyan and N. S. Bortnikov, Dokl. Akad. Nauk, Vol. 416, 2007, pp. 247-249.

[4] A. I. Gusev and A. A. Rempel, "Nanocrystal Materials," Fizmatlit, Moscow, 2001.

[5] T. L. Hill, "Thermodynamics of Small Systems," Benjamin, New York, 1963.

[6] P. Barret, "Reaction Kinetics in Heterogeneous Chemical Systems," Elsevier, New York, 1975.

[7] D. A. Frank -Kamenetskii, "Diffusion and Heat Transfer in Chemical Kinetics," Nauka, Moscow, 1967.

[8] N. M. Emanuel and D. G. Knorre, "Chemical Kinetics," Vyshaya Shkola, Moscow, 1969.

[9] V. N. Parmon, Dokl. Akad. Nauk, Vol. 413, 2007, pp. 53-59.

[10] G. O. Piloyan, "Introduction to the Theory of Thermal Analysis," Nauka, Moscow, 1964.

[11] R. L. Reed, L. Weber and B. S. Gottfried, Industrial \& Engineering Chemistry Fundamentals, Vol. 4, 1965, pp. 3846. doi:10.1021/i160013a006

[12] H. E. Kissenger, Analytical Chemistry, Vol. 29, 1957, pp. 1702-1706.

[13] X. Bokhimi, J. A. T. Antonio, M. L. Guzman-Castillo, et al., Journal of Solid State Chemistry, Vol. 161, 2001, pp. 319-326. doi:10.1006/jssc.2001.9320

[14] C. Sarda, F. Mathieu, A. Vajpei and A. Rousset, Journal of Thermal Analysis, Vol. 32, 1987, pp. 865-873. doi:10.1007/BF01913772

[15] J. Majlan, A. Navrotsky and W. H. Casey, Clays Clay Minerals, Vol. 48, 2000, pp. 699-707.

[16] M. I. Gol'dshtein, V. S. Litvinov and B. M. Bronfin, "Metallophysics of High Strength Alloys," Metallurgiya, Moscow, 1986

[17] I. N. Frantsevich, F. F. Voronov and S. A. Bakuta, "Handbook on Elastic Constants and Moduli of Elasticity for Metals and Nonmetals," Naukova Dumka, Kiev, 1982.

[18] S. Clark Jr., "Handbook on Physical Constants," Geological Society of America, New York, 1966 (Mir, Moscow, 1969).

[19] A. Navrotsky, Proceedings of National Academy of Science of the USA, Vol. 101, 2004, pp. 12096-12101. doi:10.1073/pnas.0404778101 